\title{
ORIGINAL
}

\section{INCIDENCIA HOSPITALARIA, TENDENCIA Y DISTRIBUCIÓN MUNICIPAL DEL CÁNCER DE PRÓSTATA EN EL ÁREA DE SALUD DE LEÓN (1996-2010)}

Vicente Martín Sánchez (1,2,3), Miguel García-Sanz (4), Lidia García-Martínez (3), Marbella del Canto Cabero (4), Francisco Campanario-Pérez (4), Paquita González Sevilla(1,5), Lorena Estévez Iglesias (6), Emiliano Honrado Franco (7), Ana Cuesta-Díaz de Rada (7), Fructuoso García Díez (4) y Gonzalo López-Abente (2,8).

(1) Grupo de Investigación en Interacciones gen-ambiente y salud. Universidad de León. León. España.

(2) CIBER Epidemiología y Salud Pública (CIBERESP). España.

(3) Área de Medicina Preventiva y Salud Pública. Departamento de Ciencias Biomédicas. Universidad de León. León. España.

(4) Servicio de Urología. Complejo Asistencial Universitario de León. León. España.

(5) Área de Fisiología. Departamento de Ciencias Biomédicas. Universidad de León. León. España.

(6) Registro Hospitalario de Tumores. Servicio de Anatomía Patológica. Complejo Asistencial Universitario de León. León. España.

(7) Servicio de Anatomía Patológica. Complejo Asistencial Universitario de León. León. España.

(8) Unidad de Epidemiología Ambiental y Cáncer. Instituto de Salud Carlos III - ISCIII. Madrid. España.

(*) La investigación fue financiada parcialmente por el Grupo 12 del CIBER Epidemiología y Salud Pública (CIBERESP). España.

Los autores declaran que no existen conflictos de intereses.

\section{RESUMEN}

Fundamentos. El cáncer de próstata $(\mathrm{CaP})$ es el de mayor incidencia entre los varones y sin embargo se conoce muy poco sobre sus factores de riesgo. El presente artículo tiene por objetivo conocer la incidencia hospitalaria, tendencia y distribución municipal del $\mathrm{CaP}$ en el área de salud de León (ASL)

Métodos. Fueron incluidos los casos nuevos de cáncer de próstata (CIE9: 185, CIE-10: C61) del registro hospitalario de tumores del Complejo Asistencial Universitario de León, entre 1996 y 2010 en sujetos con residencia en el ASL. Se calcularon las incidencias hospitalarias brutas trienales y ajustadas a población mundial y europea. Como denominador se utilizaron los datos del Instituto Nacional de Estadística de población desagregada por grupos quinquenales de edad de residentes en municipios del ASL. Para el análisis de la distribución espacial se estimaron los riesgos relativos (RR) municipales suavizados mediante el ajuste del modelo de Besag, York y Mollié y sus probabilidades posteriores de que los RR fuesen $>1$ (PP), utilizando métodos bayesianos.

Resultados: Se incluyeron 3.366 casos. Las tasas estandarizadas con población europea ascendieron de 30,3 (1996-98) a 119,0 (2008-2010) casos nuevos por 100.000 hombres. El número de casos órgano-confinados pasó de 281 (1999-2001) a 999 (2008-2010). Las determinaciones de PSA ascendieron de 30.985 (1999-2001) a 117.396 (2008-2010).

Conclusiones: Se observó un gran incremento de la frecuencia de $\mathrm{CaP}$ a expensas de los casos órgano-confinados, que correlacionan muy bien con las determinaciones de PSA llevadas a cabo en el ASL. No hubo diferencias de interés en la distribución municipal de las incidencias.

Palabras clave: Cáncer de próstata.Estudio de incidencias.Tendencias. Sistema de información geográfico.

Correspondencia

Vicente Martín

Grupo de Investigación en Interacciones Gen-Ambiente y Salud.

Facultad de Ciencias de la Salud. Universidad de León.

Campus de Vegazana s/n.

24071 LEÓN

Teléfono y fax: 987293110

vmars@unileon.es

DOI:

\section{ABSTRACT}

Hospital incidence, Trends and Municipal Distribution of Prostate Cancer in Health Area of León, Spain (1996-2010)

Background: Prostate cancer (PC) is the most prevalent among men and yet its risk factors are little known. This article aims to determine the hospital incidence, trend and municipal distribution of PC in Health Area of León (HAL).

Methods: We included new cases of prostate cancer (ICD-9: 185, ICD10: C61) enrolled in the Hospital Tumor Registry of the Complejo Asistencial Universitario de León, between 1996 to 2010 with residence in HAL. We calculated crude triennial hospital incidences and adjusted at global and European population. As denominator we used the INE population data disaggregated by five-year age groups of residents in municipalities of the HAL. To analyze the spatial distribution, we estimated municipal relative risks (RR) smoothed by fitting the Besag, York and Mollié model and the posterior probability (PP) of RR $>1$ using Bayesian methods.

Results: 3,366 cases were included. Standardized rates at European population amounted of 30.3 (1996-98) to 119.0 (2008-2010) new cases per $100,000 \mathrm{men}$. The number of organ-confined cases were increased from 281 (1999-2001) to 999 (2008-2010). PSA determinations amounted from 30,985 (1999-2001) to 117396 (2008-2010).

Conclusions: A great increase was observed in the frequency of $\mathrm{PC}$ at the expense of organ-confined cases which correlate very well with PSA determinations performed in HAL. There were no differences of interest in the municipal distribution incidences.

Keywords: Prostate cancer. Incidence study. Trends. Geographic information system. 


\section{INTRODUCCIÓN}

El cáncer de próstata $(\mathrm{CaP})$ es la segunda neoplasia más frecuente en el varón por incidencia y la quinta por causa de muerte a nivel mundial, estimándose en mas de $1,1 \mathrm{mi}$ llones los nuevos casos y en más de 300.000 las muertes en $2012^{1}$. La incidencia de este tumor presenta una gran variabilidad geográfica, dándose la más elevada en las zonas más desarrolladas (Australia, Nueva Zelanda, EE.UU y Europa del Oeste) aunque la mortalidad es más frecuente en el Caribe y algunas zonas de África. En el caso de la Unión Europea (UE-28) el CaP es el primer tumor en incidencia en los varones y el tercero en mortalidad, con más de 360.000 casos nuevos y 72.000 muertes estimadas en $2012^{1}$. Nuestro país presenta una tasa de incidencia intermedia y de las más bajas en mortalidad en el contexto europeo, habiéndose producido 6.038 defunciones en 2012 y estimándose en 28.000 los casos nuevos ${ }^{1}$. En nuestro país se observa un marcado incremento en la incidencia desde el uso extendido del antígeno prostático específico (PSA) mientras que la mortalidad está disminuyendo un $3,6 \%$ anual desde $1998^{2,3}$.

A pesar de su importancia para la salud pública, son pocos los factores de riesgo bien establecidos para el CaP y ninguno modificable (edad, raza y antecedentes familiares), si bien se considera que el $\mathrm{CaP}$ tiene un carácter multifactorial dónde las interacciones entre la carga genética, el estilo de vida y los factores endocrinos están en el origen y la gravedad y/o agresividad de este tumor ${ }^{4-7}$. También se ha advertido sobre la posible asociación de este tumor con ciertas ocupaciones, en especial las que favorecen el contacto con pesticidas $^{6}$. Sin embargo su patrón geográfico de distribución tumor es poco informativo y apenas se ha modificado en los últimos decenios?

Por todo ello el estudio tuvo por objetivo conocer la incidencia hospitalaria, su tendencia y la distribución geográfica del $\mathrm{CaP}$ en el área de salud de León.

\section{MATERIAL Y MÉTODOS}

Se diseñó un estudio observacional descriptivo utilizando los datos del Registro Hospitalario de Tumores del Complejo Asistencial Universitario de León (CAULE), hospital de referencia del Área de Salud de León, la cual tiene una población de unos 350.000 habitantes. Este registro se guía por el Manual de procedimientos en los Registros Hospitalarios de Tumores de la Sociedad Española de Anatomía Patológica ${ }^{8}$ e incorpora información desde 1993, codificando los diagnósticos según la Clasificación Internacional de Enfermedades (CIE-9, CIE-10).

En el estudio se incluyeron todos los diagnósticos registrados de neoplasia maligna de próstata (CIE-9: 185, CIE-10: C61) desde el 1 de enero de 1996 hasta el 31 de diciembre de 2010 , en varones con residencia en cualquier municipio del área de salud de León, según la tarjeta sanitaria. Al objeto de evitar duplicidades y evitar errores en el domicilio de residencia, se comprobaron individualmente todos los casos seleccionados y se revisaron las coincidencias en fecha de nacimiento, número de historia o documento nacional de identidad. Así mismo se comprobó la coincidencia entre la residencia reflejada en la ficha de admisión con la referida en la tarjeta sanitaria.

Los tumores se diferenciaron en locales, regionales, metastásicos y desconocidos, atendiendo a los criterios de la clasificación TNM de $2009^{9}$.

Para el cálculo de las tasas se utilizó como denominador la población registrada por el Instituto Nacional de Estadística por municipios, desagregada por sexo y edad (grupos quinquenales de edad). Se calcularon las tasas estandarizadas con la población mundial y con la europea. Con el fin de reducir la variabilidad anual se calcularon las tasas brutas y estandarizadas por trienios (de 1996 a 1998, de 1999 a 2001, de 2002 a 2004, de 2005 a 2007 y de 2008 a 2010). 
A través de los registros del Servicio de Análisis Clínicos se obtuvieron las cifras globales de peticiones procesadas de la determinación de antígenos prostático específico (PSA) desde el año 1999 hasta el 2010, agrupándose posteriormente en los trienios anteriormente descritos.

Las tasas de mortalidad fueron obtenidas del servidor interactivo de información epidemiológica del Centro Nacional de Epidemiología ${ }^{10}$. Debería ser la referencia número 10 .

Se estudió la distribución por municipios calculándose las razones estandarizadas de incidencia hospitalaria, definidas como el cociente entre los casos observados y los esperados. Para el cálculo de los casos esperados se tomaron como referencia las tasas específicas de cada grupo de edad para el conjunto del Área de Salud. Para cada municipio se efectuó una estimación de los riesgos relativos (RR) municipales suavizados mediante el ajuste del modelo de Besag-York y Mollié (BYM) ${ }^{11}$. Este modelo se ha popularizado como la forma estándar para la elaboración de mapas de enfermedades, ya que resuelve de una forma muy eficiente el problema de las "áreas pequeñas". Se basa en el ajuste de modelos espaciales de Poisson con los casos observados como variable dependiente, los esperados como offset y dos términos de efectos aleatorios: 1) uno que es independiente para cada municipio y que representa la heterogeneidad municipal y 2) un término espacial que permite que cada municipio comparta su información promediándola con la de sus municipios vecinos, utilizando el criterio vecindad como adyacencia de contornos municipales. Estos riesgos relativos (RR) suavizados y sus probabilidades posteriores (PP) de que los RR fueran mayores de $1^{12}$ se estimaron mediante métodos bayesianos empleando integrated nested Laplace approximation utilizando (RINLA) $)^{13}$ con el programa $\mathrm{R}^{14}$.

\section{RESULTADOS}

Se incluyó a 3.366 varones residentes en municipios del Área de Salud de León diagnosticados entre el 1 de enero de 1996 y el 31 de diciembre de 2010. Del total, $3.115(92,6 \%)$ de los casos fueron diagnosticados mediante biopsia.

La edad de los sujetos varió desde los 31 años hasta los 110 , con una edad media en los tumores localizados de 76,4 años, en los

\section{Figura 1}

Tasas de incidencia de cáncer de próstata ajustadas a población europea por 100.000 hombres en el Área de Salud de León (1996-2010). Grupos de edad en años cumplidos y tasas específicas en cada uno de ellos

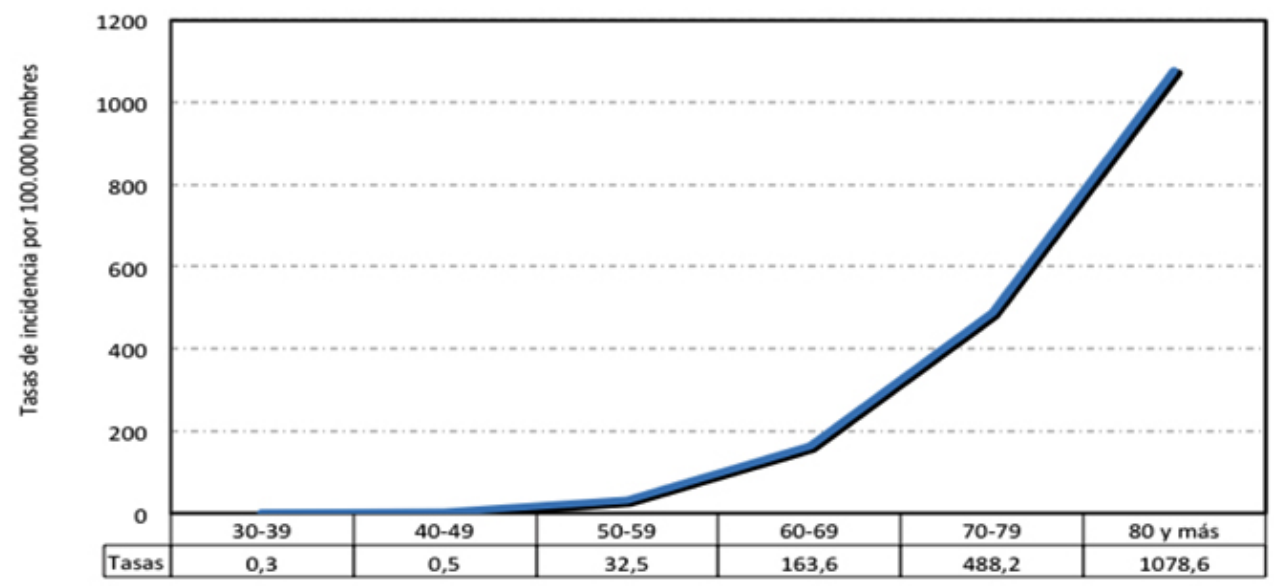




\section{Tabla 1}

Distribución de los casos de cáncer de próstata, población, tasas brutas y tasas ajustadas según trienio en el área de salud de León (1996-2010)

\begin{tabular}{|c|c|c|c|c|c|}
\hline \multirow{2}{*}{ Trienio } & \multirow{2}{*}{ Casos } & \multirow{2}{*}{ Población } & \multicolumn{3}{|c|}{ Tasas por 100.000 } \\
\cline { 3 - 5 } & & 526.329 & Bruta & $\mathrm{EPE}^{1}$ & $\mathrm{EPM}^{2}$ \\
\hline $1996-98$ & 290 & 515.405 & 91,1 & 30,3 & 16,6 \\
\hline $1999-01$ & 472 & 508.107 & 117,5 & 44,6 & 24,3 \\
\hline $2002-04$ & 597 & 512.088 & 168,7 & 78,5 & 29,6 \\
\hline $\mathbf{2 0 0 5 - 0 7}$ & 864 & 516.266 & 221,4 & 119,0 & 77,6 \\
\hline $2008-10$ & 1143 & \multicolumn{4}{|l}{} \\
\hline${ }^{1}$ EPE: a población europea; ${ }^{2} \mathrm{EPM}$ a población mundial. \\
\hline
\end{tabular}

\section{Figura 2}

Distribución de las tasas de incidencia y mortalidad, ajustadas a población europea y determinaciones de PSA llevadas a cabo en el área de salud de León por trienio

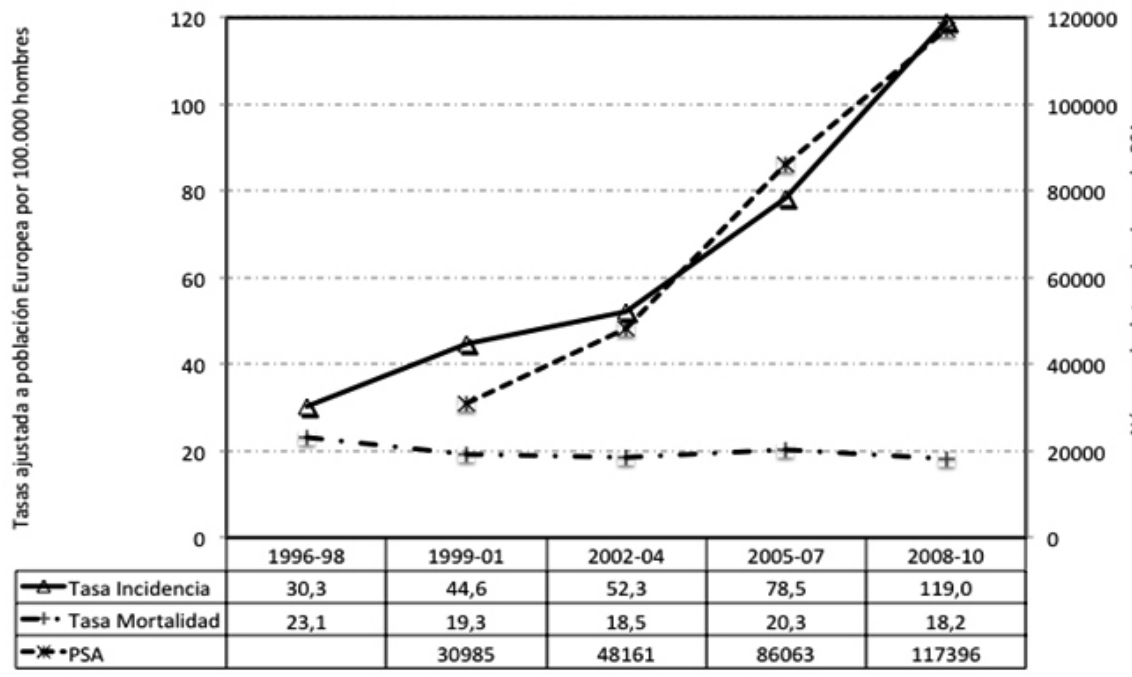

\section{Tabla 2}

Distribución de los casos de cáncer de de próstata según estadío clínico y trienio de diagnóstico en el área de salud de León (1996-2010)

\begin{tabular}{|c|c|c|c|c|c|c|c|c|c|c|}
\hline \multirow{3}{*}{ Estadío } & \multicolumn{10}{|c|}{ Trienio } \\
\hline & \multicolumn{2}{|c|}{ 1996-98 } & \multicolumn{2}{|c|}{ 1999-01 } & \multicolumn{2}{|c|}{ 2002-04 } & \multicolumn{2}{|c|}{ 2005-07 } & \multicolumn{2}{|c|}{$2008-10$} \\
\hline & $\mathrm{n}$ & $\%$ & $\mathrm{n}$ & $\%$ & $\mathrm{n}$ & $\%$ & $\mathrm{n}$ & $\%$ & $\mathrm{n}$ & $\%$ \\
\hline Localizado & 116 & 40,0 & 281 & 59,5 & 359 & 60,1 & 597 & 69,1 & 999 & 87,4 \\
\hline Regional & 25 & 8,6 & 29 & 6,1 & 23 & 3,9 & 12 & 1,4 & 77 & 6,7 \\
\hline Metastásico & 71 & 24,5 & 69 & 14,6 & 89 & 14,9 & 90 & 10,4 & 58 & 5,1 \\
\hline Desconocido & 78 & 26,9 & 93 & 19,7 & 126 & 21,1 & 165 & 19,1 & 9 & 0,8 \\
\hline Total & \multicolumn{2}{|c|}{290} & \multicolumn{2}{|c|}{472} & \multicolumn{2}{|c|}{597} & \multicolumn{2}{|c|}{864} & \multicolumn{2}{|c|}{1.143} \\
\hline
\end{tabular}


Figura 3

Distribución de los riesgos relativos municipales suavizados y la probabilidad postprueba
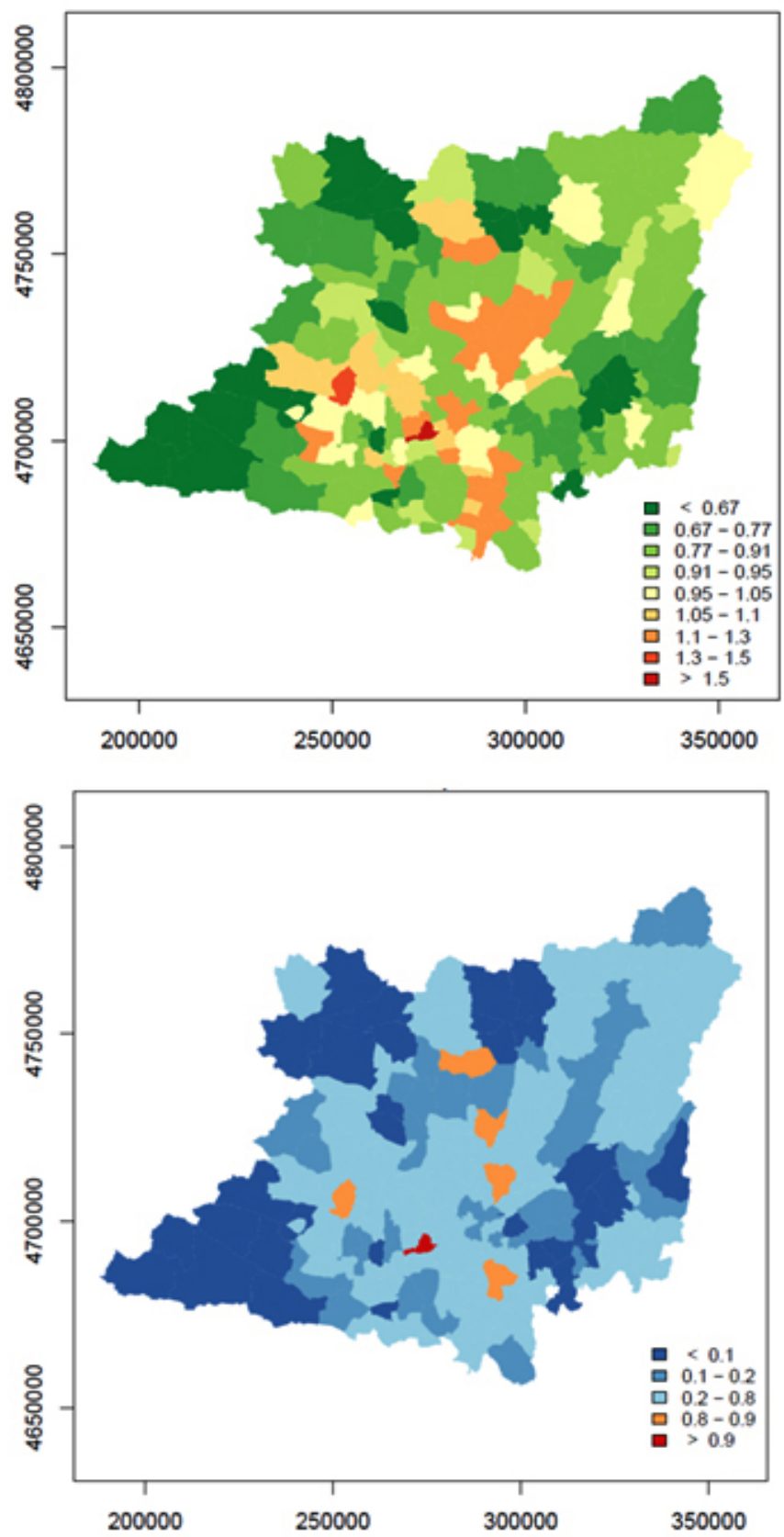
regionales de 75,1 años, en los metastásicos de 78,2 y en los desconocidos de 83,0 años. En la figura 1 se pueden observar las tasas por grupos de edad.

En la tabla 1 se puede observar, por trienios, la distribución de los casos y tasas, brutas y ajustadas. De menos de 100 casos anuales y una tasa ajustada con la población europea de 30,3 casos nuevos por $10^{-5}$ hombres y año en el trienio 1996-1998 se pasó a 380 casos al año y unas tasas ajustadas con población europea de 119 casos nuevos por $10^{-5}$ hombres y año en el trienio 2008-2010.

Como se puede observar en la figura 2, el incremento observado en las tasas de incidencia hospitalaria se correlacionó de una manera muy clara con el número de determinaciones de PSA llevadas a cabo en el área de salud, de manera que por cada cien determinaciones se registró un caso de CaP. Por el contrario, las tasas de mortalidad de la provincia mostraron una tendencia ligeramente decreciente, desde 23,1 muertes por 100.000 hombres y año en el trienio 1996-1998 a 18,2 muertes por 100.000 hombres y año en el trienio 20082010, es decir, un descenso del $21 \%$ entre el primer y el último trienio.

En la tabla 2 se puede observar cómo el incremento en el número de casos fue a costa de los casos localizados, desde 38,7 casos al año en el primer trienio se alcanzaron los 333 anuales durante el último. Del mismo modo se puede observar cómo el número de casos metastásicos se mantuvo estable a lo largo de los quince años, en torno a 70 casos en cada trienio.

Con relación a la distribución geográfica de los casos se observaron cuatro zonas geográficas con un mayor riesgo: zona de la Sobarriba - Porma, Esla- Páramo, TuertoÓrbigo y Bernesga, destacando Santa María del Páramo donde se observó un riesgo relativo de 1,51 y una PP de 0,94 (figura 3).

\section{DISCUSIÓN}

En el Área de salud de León se observa un notable incremento tanto del número de casos de CaP como de la tasa de incidencia, que prácticamente se han cuadruplicado desde el trienio 1996-1998 al 2008-2010. Este incremento se produce fundamentalmente a expensas de los casos órgano-confinados y se correlaciona muy bien con el incremento del número de determinaciones analíticas de PSA llevadas a cabo en el Área. Este incremento en la incidencia no se ve acompañado de un aumento en la mortalidad, ya que la tasa de mortalidad por $\mathrm{CaP}$ presenta una ligera tendencia descendente en la provincia.

Estos hallazgos son congruentes con lo observado por otros autores, que atribuyen el importante incremento de la incidencia al uso extendido de la prueba de $\mathrm{PSA}^{2,15,16}$. Como es sabido, la extensión de la determinación de PSA como prueba de cribado es un tema controvertido, ya que al incrementar el sobrediagnóstico no se ve un efecto claro sobre la mortalidad específica y tiene efectos negativos sobre la calidad de vida de los pacientes $^{16,17}$.

El incremento de la incidencia observado en nuestro área de salud es muy superior a lo indicado por otros autores. En los registros españoles se ha informado de un incremento anual en España del 7,3\% entre 1990 y $2004^{2}$ y en Cataluña del 6,3\% entre 1993 y $2007^{18}$. Una posible explicación a este incremento, por encima de lo publicado por otros autores, es el retraso en la realización de las pruebas de PSA como cribado oportunista en nuestro área de salud. Esta explicación se ve reforzada por el hecho de que en el quinquenio 1995-99 la incidencia por 100.000 hombres, en los registros españoles oscilaba entre el 40,5 de Granada y el 83,7 de Canarias, con una incidencia global media de 63,1. Estas cifras son muy superiores a las encontradas en nuestro registro para esas fechas $(30,3)$. Del mismo modo, en el quinquenio 20002004 la tasa de los registros españoles osci- 
laba entre el más bajo de Granada $(49,1)$ y el más elevado del País Vasco $(104,8)$ y la incidencia global media era de 86,6 mientras que en nuestra área de salud se encontraba en la zona baja de la tabla (52,3). Finalmente, nuestras cifras de incidencia en el último trienio (119) son incluso superiores a las comunicadas para el año 2012 para España (96,8 nuevos casos por 100.000 hombres) o las publicadas por Cózar para el año 2010 $(70,8)^{17}$.

La tasa de mortalidad en la provincia es similar a la comunicada para España entre 2002 y $2006(19,8)^{19}$ ó en 2012. Lo mismo sucede con la tendencia, nuestros datos son también consistentes con los de la literatura, se observa un moderado descenso de la misma, debido fundamentalmente a las mejoras tanto en el diagnóstico como en el tratamiento, lo que se traduce en unas elevadas tasas de supervivencia a los cinco años. Así, la supervivencia a los 5 años se incrementó desde el 75,3\% durante el período 1995-1999 hasta el $85,0 \%$ durante el período 2000-2003, según Marcos-Grajera ${ }^{20}$ o entre el 73,7\% en el quinquenio $1995-1999$ al $87,1 \%$ en el quinquenio 2005-2009 según el estudio Concord- $2^{21}$. Sin embargo, el descenso observado es inferior al publicado para España entre 1997 y $2006(-3,7 \% \text { anual })^{2}$ o Cataluña $(-2,9 \%)^{18}$ y más cercano al descenso observado en Andalucía para ese período (-1,8\%) que el observado en Navarra $(-8,8 \%)$.

Con relación a las características de los casos, se pone de manifiesto lo observado en otros estudios, en los que el incremento de casos es achacable al incremento de los casos localizados. También se observa cómo el número de casos metastásicos se mantiene relativamente estable. El porcentaje de pacientes con enfermedad localizada en el último trienio $(87,4 \%)$ es muy similar al publicado por Cózar et al. ${ }^{17}$ y ligeramente superior al encontrado en Estados Unidos $(81 \%)^{22}$. El porcentaje de casos metastásicos es similar al de los estudios anteriores del $4 \%$ y en nuestro caso del $5 \%{ }^{17,22}$.
Con relación al patrón municipal observado no es particularmente notable, tal y como sucede con el del mapa de mortalidad elaborado por López Abente a nivel nacional $^{7}$. Esto es explicable porque ninguno de los factores de riesgo descritos se relaciona territorialmente (susceptibilidad genética, las hormonas, la dieta o el consumo de productos lácteos). En nuestro caso destaca la mayor incidencia detectada en cuatro zonas con un mayor riesgo: zona de la SobarribaPorma, Esla-Páramo, Tuerto-Órbigo y Bernesga, zonas dedicadas al primer sector (agricultura), si bien las diferencias observadas no pueden ser consideradas estadísticamente significativas ni relevantes. Diversos estudios han relacionado la incidencia de cáncer de próstata con la exposición a pesticidas y otros componentes a los que los agricultores y granjeros tienen contacto, como polvos orgánicos e inorgánicos, combustiones, fertilizantes químicos, etcétera. La relación de estos factores etiológicos con el cáncer de próstata es débil debido a la gran variedad de sustancias utilizadas y a la difícil discriminación de la diferencia en la exposición a estas sustancias entre quienes presentaron carcinoma de próstata y la población general.

La principal limitación de nuestros resultados radica en que la fuente de casos es un registro hospitalario y no un registro poblacional. Los registros de base poblacional recogen de forma sistemática y continuada todos los casos que van apareciendo en toda la población y utilizan sistemas de control de calidad que incluyen la exhaustividad y la exactitud. En el caso de los registros hospitalarios su objetivo y finalidad es evaluar la calidad de la asistencia a los pacientes diagnosticados de cáncer y/o atendidos en el hospital, pero en algunas circunstancias son también de utilidad para conocer la frecuencia anual de casos y su tendencia en función de las diferentes características de los pacientes ${ }^{8}$. Los registros hospitalarios, en comparación con los poblacionales, habitualmente subestiman el número de casos, en 
mayor o menor medida, en función del nivel de cobertura del hospital y del acceso de la población de referencia ${ }^{23}$. Ello viene dado por diversos factores como el número de hospitales en el área y el grado de cobertura del Servicio de Salud. En nuestro caso, el CAULE, es el único hospital del área de salud de León y el grado de cobertura del Servicio Castellano y Leonés de Salud (Sacyl) es superior al $94 \% 0^{24,25}$. Todo ello permite pensar que son pocos los cánceres de próstata que no son diagnosticados o atendidos en el mismo. En cualquier caso, las tasas observadas sí nos pueden dar una idea de la incidencia mínima del área y de la tendencia temporal de la misma.

A modo de resumen podemos destacar que se aprecia un aumento exponencial en el diagnóstico de cáncer de próstata en el Área de Salud de León desde 1996 a 2010, a expensas fundamentalmente de tumores órgano-confinados. Dicho aumento puede corresponder a un mayor diagnóstico en relación con la difusión de la determinación del PSA en la población masculina. La tasa de mortalidad es acorde con la informada en nuestro país así como su tendencia ligeramente descendente. Diferentes zonas de la provincia destacan por una incidencia más acusada de esta patología, posiblemente relacionada con la ocupación laboral (primer sector, agricultura) sin poder determinarse dicha relación en el presente estudio.

\section{AGRADECIMIENTOS}

A la Dra. Teresa Ribas por sus consejos y por mantener vivo el registro hospitalario de tumores del Complejo Asistencial Universitario de León.

\section{BIBLIOGRAFÍA}

1. Ferlay J, Soerjomataram I, Ervik M, Dikshit R, Eser S, Mathers C, et al. GLOBOCAN 2012 v1.0, Cancer Incidence and Mortality Worldwide: IARC CancerBase No. 11. Lyon: International Agency for Research on Cancer; 2013. Disponible en: http://globocan.iarc.fr, accessed on 14/agosto/2014.
2. Larrañaga N, Galceran J, Ardanaz E, Franch P, Navarro C, Sánchez MJ et al. Prostate cancer incidence trends in Spain before and during the prostate-specific antigen era: impact on mortality. Ann Oncol. 2010; S3:iii83-89.

3. López-Abente G, Mispireta S, Pollán M. Breast and prostate cancer: an analysis of common epidemiological features in mortality trends in Spain. BMC Cancer. 2014; $14: 874$

4. Discacciati A, Wolk A. Lifestyle and dietary factors in prostate cancer prevention. Recent Results Cancer Res. 2014; 202:27-37.

5. Schaid DJ, Stanford JL, McDonnell SK, Suuriniemi M, McIntosh L, Karyadi DM, et al. Genome-wide linkage scan of prostate cancer Gleason score and confirmation of chromosome 19q. Hum Genet. 2007; 121: 729735 .

6. Alavanja MC, Bonner MR. Occupational pesticide exposures and cancer risk: a review. J Toxicol Environ Health B Crit Rev. 2012;15(4):238-63.

7. López-Abente G, Aragonés N, Pérez-Gómez B, Pollán M, García-Pérez J, Ramis R, et al. Time trends in municipal distribution patterns of cancer mortality in Spain. BMC Cancer. 2014;14:535.

8. Grupo Nacional Multidisciplinar de Registros Hospitalarios de Tumores. Manual de procedimientos en los Registros Hospitalarios de Tumores. Málaga: Sociedad Española de Anatomía Patológica; 2007.

9. Sobin LH, Gospodariwicz M, Wittekind C (editors). TNM classification of malignant tumors. UICC International Union Against Cancer. 7th ed. Wiley-Blackwell, 2009.

10. servidor interactivo de información epidemiológica (Ariadna). Madrid: Instituto de Salud Carlos III. Disponible en: http://ariadna.cne.isciii.es/

11. Besag J, York J, Mollié A. Bayesian image restoration, with two applications in spatial statistics.Ann Inst Stat Math. 1991; 43:1-59.

12. Richardson S, Thomson A, Best N, Elliott P. Interpreting posterior relative risk estimates in disease-mapping studies. Environ Health Perspect 2004; 112(9): 1016-25.

13. Rue H, Martino S, Chopin N. Approximate Bayesian Inference for Latent Gaussian Models Using Integrated Nested Laplace Approximations. Journal of the Royal Statistical Society: Series B. 2009; 61: 319-392.

14. R Development Core Team. R: A language and environment for statistical computing. Disponible en: http:// www.R-project.org/. 
15. Neppl-Huber C, Zappa M, Coebergh JW, Rapiti E, Rachtan J, Holleczek B, et al. Changes in incidence, survival and mortality of prostate cancer in Europe and the United States in the PSA era: additional diagnoses and avoided deaths. Ann Oncol. 2012;23:1325-34.

16. Kim EH, Andriole GL. Prostate-specific antigenbased screening: controversy and guidelines. BMC Med. 2015;13:61.

17. Cózar JM, Miñana B, Gómez-Veiga F, RodríguezAntolín A, Villavicencio H, Cantalapiedra A, et al. Registro nacional de cáncer de próstata 2010 en España. Actas Urol Esp. 2013;37(1):12-19.

18. Cleries R, Esteban L, Borrás J, Marcos-Gragera R, Freitas A, Carulla $\mathrm{M}$ et al. Time trends of cancer incidence and mortality in Catalonia during 1993-2007. Clin Transl Oncol. 2014; 16:18-28.

19. Cabanes A, Pérez-Gómez B, Aragonés N, Pollán M, López-Abente G. La situación del cáncer en España, 1975-2006. Madrid: Instituto de Salud Carlos III;2009.

20. Marcos-Gragera R, Salmerón D, Izarzugaza I, Ardanaz E, Serdà BC, Larrañaga N. Trends in prostate cancer survival in Spain: results from population-based cancer registries. Clin Transl Oncol. 2012; 14:458-464.

21. Allemani C, Weir HK, Carreira H, Harewood R, Spika D, Wang XS, et al. Global surveillance of cáncer survival 1995-2009: analysis of individual data for 2566887 patients from 279 population-based registries in 67 countries (CONCORD-2). Lancet. 2015;385:9771010 .

22. Howlader N, Noone AM, Krapcho M, Neyman N, Aminou R, Waldron W, et al. SEER Cancer Statistics Review, 1975-2008. MD: National Cancer Institute Bethesda; 2011.

23. Bernal-Delgado EE, Martos C, Martínez N, Chirlaque MD, Márquez M, Navarro C, et al. Is hospital discharge administrative data an appropriate source of information for cancer registries purposes? Some insights from four Spanish registries. BMC Health Serv Res. 2010 ;10:9.

24. Naveiro JC, Peral A, Díez A, González FA, Burón JL. Incidencia y riesgo de cáncer en el área sanitaria de León. Oncología. 2001; 24:21-28.

25. García Martínez L, Pollán Santamaría M, LópezAbente G, Sánchez Jacob MM, García Palomo A, González Martínez R, et al. Tendencia y distribución municipal de la incidencia de cáncer de mama en el área de salud de León (1996-2010). Rev Esp Salud Pública. 2014; 88:1-9. 